\title{
ON THE STIEFEL-WHITNEY CLASSES OF \\ A MANIFOLD. II
}

\author{
W. S. MASSEY 1
}

1. Introduction. This note is a complement to a previous paper of the same title [1]. In that paper it was proved that certain StiefelWhitney classes or dual Stiefel-Whitney classes modulo 2 of a differentiable manifold always vanished. In the present note, analogous theorems are proved about the integral Stiefel-Whitney classes of a manifold.

The following convention regarding notation will be followed consistently. The mod 2 Stiefel-Whitney classes are denoted by lowercase letters, $w_{i}$, while the integral Stiefel-Whitney classes are denoted by capital letters, $W_{i}$; the subscript denotes the dimension. A bar over the appropriate symbol denotes the dual Stiefel-Whitney class, integral or mod 2, thus: $\bar{W}_{i}, \bar{w}_{i}$. Of course the integral classes are only defined in odd dimensions.

Let $M^{n}$ denote a compact, connected, orientable, differentiable $n$ manifold. We will prove the following three theorems about its integral Stiefel-Whitney classes:

ThEOREM 1. If $n$ is even, $\bar{W}_{n-1}=0$.

THEOREM 2. If $n$ is even, $W_{n-1}=0$.

THEOREM 3. If $n \equiv 3 \bmod 4, W_{n-2}=0$.

Note that Theorem 1 implies Corollary 1 to Theorem I of [1] in the orientable case. Similarly, Theorem 2 implies Theorem II of [1], and Theorem 3 represents a strengthening of part of the conclusion of Theorem III of [1].

The main interest in Theorem 1 stems from the fact that A. Haefliger and M. Hirsch have recently proved [3] that any (compact, orientable, differentiable) $M^{n}, n>4$, is differentiably imbeddable in $R^{2 n-1}$ if and only if $\bar{W}_{n-1}=0$ for $n$ even and $\bar{w}_{n-1}=0$ for $n$ odd. Thus it follows from Theorem 1 above and Corollary 1 to Theorem I of [1] that such an $M^{n}$ is always differentiably imbeddable in $R^{2 n-1}$ for $n>4$.

An interesting application of Theorem 2 is to 8-dimensional manifolds. According to $\left[2\right.$, p. 170], for any compact, orientable $M^{8}$, $W_{5}=0$; by Theorem $2, W_{7}=0$. Thus $W_{3}$ is the only integral Stiefel-

Received by the editors November 16, 1961.

1 During the preparation of this note the author was partially supported by N.S.F. Grant G-18895. 
Whitney class of an 8-dimensional manifold which can be nonzero. It is known that $W_{3}$ is the first obstruction to the existence of an almost complex structure on $M^{8}$, and if $W_{3}=0$, then $W_{7}$ is the second obstruction. Therefore if we remove a single point from any compact, orientable $M^{8}$ for which $W_{3}=0$, the resulting noncompact manifold admits an almost-complex structure.

Note also that Theorem 2 above and Theorem III of [1] imply that the first obstruction to defining a field of tangent 2 -frames on a compact, orientable $n$-manifold always vanishes provided $n$ - $1 \bmod 4$. This raises the question of determining the second obstruction to such a field.

2. A lemma. Assume that $M^{n}$ is a compact, connected orientable $n$-manifold. Let $T^{q}$ denote the torsion subgroup of $H^{q}\left(M^{n}, \boldsymbol{Z}\right)$. Let $p$ be a prime number, and

$$
H^{q}(M, Z) \stackrel{p}{\rightarrow} H^{q}(M, Z) \stackrel{r}{\rightarrow} H^{q}\left(M, Z_{p}\right) \stackrel{\delta^{*}}{\rightarrow} H^{q+1}(M, Z)
$$

be the exact sequence associated with the coefficient sequence

$$
0 \rightarrow \boldsymbol{Z} \stackrel{p}{\rightarrow} \boldsymbol{Z} \rightarrow \boldsymbol{Z}_{p} \rightarrow 0
$$

The cup product is a bilinear form

$$
\cup: H^{q}\left(M^{n}, Z_{p}\right) \times H^{n-q}\left(M^{n}, Z_{p}\right) \rightarrow H^{n}\left(M^{n}, Z_{p}\right) \approx Z_{p}
$$

According to the Poincaré Duality Theorem, this bilinear form is nondegenerate.

LEMma 1. For any $q, r\left(T^{n-q}\right)$ is the annihilator of $r\left[H^{q}(M, Z)\right]$.

Proof. It is clear that $r\left(T^{n-q}\right)$ is contained in the annihilator of $r\left[H^{q}(M, Z)\right]$. We will complete the proof by showing that $r\left(T^{q}\right)$ has the same rank (as a vector space over $\boldsymbol{Z}_{p}$ ) as the annihilator of $r\left(H^{q}\right)$. For this purpose we introduce the following notation:

$b_{i}=i$ th Betti no. of $M=\operatorname{rank}$ of $H^{i}(M, \boldsymbol{Z})$.

$c_{i}=$ number of cyclic summands in the $p$-primary component of $T^{i}$ (i.e., the $p$-primary component of $T^{i}$ is the direct sum of $c_{i}$ cyclic subgroups).

According to the Poincaré Duality Theorem, $b_{i}=b_{n-i}$ and $c_{i}=c_{n-i+1}$. Consideration of the exact sequence (S) shows that the rank of the vector space $H^{i}\left(M, \boldsymbol{Z}_{p}\right)$ is $b_{i}+c_{i}+c_{\imath+1}$ while the ranks of the subspaces $r\left(H^{i}\right)$ and $r\left(T^{i}\right)$ are $b_{i}+c_{i}$ and $c_{i}$, respectively. Therefore the rank of the annihilator of $r\left(H^{q}\right)$ is 


$$
\begin{aligned}
& \left(b_{n-q}+c_{n-q}+c_{n-q+1}\right)-\left(b_{q}+c_{q}\right) \\
& \quad=\left(b_{q}+c_{n-q}+c_{q}\right)-\left(b_{q}+c_{q}\right)=c_{n-q}
\end{aligned}
$$

which is precisely the rank of the subspace $r\left(T^{n-q}\right)$, as was to be proved.

REMARK. This lemma is apparently well known; see [2, p. 169].

3. Proof of Theorem 1. We will use the exact sequence (S) and the preceding lemma for the case $p=2$. It is well known that

$$
\bar{W}_{i+1}=\delta^{*}\left(\bar{v}_{i}\right),(i \text { even }) ;
$$

in fact, this equation may be taken as the definition of $\bar{W}_{i+1}$. Hence by exactness of $(S)$, to prove $\bar{W}_{n-1}=0$, it suffices to prove that $\bar{w}_{n-2} \in r\left(H^{n-2}\right)$. By the preceding lemma, this is equivalent to proving that $\bar{w}_{n-2}$ annihilates the subspace $r\left(T^{2}\right) \subset H^{2}\left(M, Z_{2}\right)$.

By Lemma 7 of [1], the homomorphism $H^{2}\left(M^{n}, \boldsymbol{Z}_{2}\right) \rightarrow H^{n}\left(M^{n}, \boldsymbol{Z}_{2}\right)$ defined by $x \rightarrow x \cdot \bar{w}_{n-2}$ is a sum of iterated Steenrod squares, which we may assume to be admissible on account of Adem's relations. By Lemma 4 of [1] we may assume that the excess of any such admissible iterated Steenrod square is 1 or 2 . We will complete the proof by showing that for $x \in r\left(T^{2}\right)$,

$$
S q^{I}(x)=0,
$$

where $S q^{I} ; H^{2}\left(M^{n}, Z_{2}\right) \rightarrow H^{n}\left(M^{n}, Z_{2}\right)$ is any admissible iterated Steenrod square of excess 1 or 2 and degree $n-2$.

In case the excess is 1 , then we must have $I=\left(2^{i}, 2^{j-1}, \cdots, 2,1\right)$ for some integer $j \geqq 0$. But in this case it is clear that $S q^{I} x=0$, for $x \in r\left(T^{2}\right)$, because

$$
S q^{1} x=r \delta^{*}(x)=0
$$

by exactness of $(\mathrm{S})$.

In case the excess is 2 , by Lemma 5 of [1] there exists an admissible iterated Steenrod square, $S q^{j}$, and a power of $2, m=2^{k}$, such that

$$
S q^{I} x=\left(S q^{J} x\right)^{m}
$$

and $J$ has excess 0 or 1 . In case $J$ has excess 0 , then

$$
S q^{I} x=x^{m}
$$

and it is obvious that $S q^{I} x=0$ for $x \in r\left(T^{2}\right)$. In case $J$ has excess 1, then $J=\left(2^{j}, 2^{j-1}, \cdots, 2,1\right)$ for some integer $j \geqq 0$ exactly as before, and $S q^{J}(x)=0$ for $x \in r\left(T^{2}\right)$ for the same reason as before. Thus in either case, $S q^{I}(x)=0$ for $x \in r\left(T^{2}\right)$, as was to be proved. 
4. Proof of Theorem 2. We will divide the proof into two cases, according as $n=4 k+2$ or $n=4 k$. In both cases, use will be made of the following lemma.

LEMma 2. For any integer $i, U_{i}^{2}$ is the reduction mod 2 of an integral cohomology class.

Proof. Here $U_{i}$ denotes the class of $\mathrm{Wu}$; the Stiefel-Whitney classes are defined in terms of the $U_{i}$ by the formula

$$
w_{j}=\sum_{i} S q^{j-i} U_{i}
$$

It is readily seen by induction on $j$ that the $U_{i}$ may be expressed as polynomials in the Steenrod squares of the $w_{j}$, and hence as polynomials in the Stiefel-Whitney classes $w_{j}$ (since any Steenrod square of a Stiefel-Whitney class may be expressed as a polynomial in the Stiefel-Whitney classes). Now it is well known that the square of any Stiefel-Whitney class, $w_{j}^{2}$, is the reduction mod 2 of an integral class; for $j$ even, it is the reduction of a Pontrjagin class, while for $j$ odd it is the reduction of $W_{j}^{2}$. Hence the square of any polynomial in the $w_{j}$ is the reduction mod 2 of an integral class. In particular, $U_{i}^{2}$ is the reduction of an integral class.

First we will prove Theorem 2 for the case $n=4 k+2$, the easiest case. In this case

$$
w_{n-2}=w_{4 k}=S q^{2 k} U_{2 k}=U_{2 k}^{2}
$$

which is the reduction mod 2 of an integral class by the lemma. Hence

$$
W_{n-1}=\delta^{*}\left(w_{n-2}\right)=0
$$

by the exactness of (S).

Next, we will prove Theorem 2 for the case $n=4 k$. In this case

$$
w_{n-2}=w_{4 k-2}=S q^{2 k-2} U_{2 k} .
$$

To prove $W_{n-1}=0$, it suffices to prove that $x \cdot w_{n-2}=0$ for any $x \in r\left(T^{2}\right)$, as in the proof of Theorem 1. To achieve this, it obviously suffices to prove that for $m=2^{q}, q \geqq 0$, and $x \in r\left(T^{2}\right)$,

$$
x^{m} S q^{2 k-2 m} U_{2 k}=x^{2 m} S q^{2 k-4 m} U_{2 k} \text {. }
$$

For, successive application of (1) with $m=1,2,4,8, \cdots$ shows that

$$
\begin{aligned}
x \cdot w_{n-2} & =x \cdot S q^{2 k-2} U_{2 k}=x^{2} \cdot S q^{2 k-4} U_{2 k} \\
& =x^{4} \cdot S q^{2 k-8} U_{2 k}=\cdots=0 .
\end{aligned}
$$


To prove (1), note first that since $S q^{1} x=0$, the only nonzero Steenrod squares of $x^{m}$ are

$$
\begin{aligned}
S q^{0} x^{m} & =x^{m}, \\
S q^{2 m} x^{m} & =x^{2 m} .
\end{aligned}
$$

Therefore by Cartan's formula,

$$
S q^{2 k-2 m}\left(x^{m} U_{2 k}\right)=x^{m} S q^{2 k-2 m} U_{2 k}+x^{2 m} S q^{2 k-4 m} U_{2 k} .
$$

But by the definition of the $U_{i}$,

$$
\begin{aligned}
S q^{2 k-2 m}\left(x^{m} U_{2 k}\right) & =U_{2 k-2 m}\left(x^{m} U_{2 k}\right) \\
& =\left(U_{2 k-2 m} x^{m}\right) U_{2 k}=S q^{2 k}\left(U_{2 k-2 m} x^{m}\right) \\
& =\left(U_{2 k-2 m} x^{m}\right)^{2}=U_{2 k-2 m}^{2} x^{2 m} .
\end{aligned}
$$

By Lemma 2, $U_{2 k-2 m}^{2}$ is the reduction $\bmod 2$ of an integral class; since $x \in r\left(T^{2}\right), U_{2 k-2 m}^{2} x^{2 m} \in r\left(T^{n}\right)$, i.e.,

$$
U_{2 k-2 m}^{2} x^{2 m}=0 \text {. }
$$

Combining (2), (3), and (4) gives (1) as desired.

5. Proof of Theorem 3. Let $n=4 k+3$; then

$$
w_{n-3}=w_{4 k}=S q^{2 k} U_{2 k}=U_{2 k}^{2}
$$

which is the reduction $\bmod 2$ of an integral class by Lemma 2 , and

$$
W_{n-2}=\delta^{*}\left(w_{n-3}\right)=0
$$

by exactness of $(\mathrm{S})$.

\section{BIBLIOGRAPHY}

1. W. S. Massey, On the Stiefel-Whitney classes of a manifold, Amer. J. Math. 82 (1960), 92-102.

2. F. Hirzebruch and H. Hopf, Felder von Flächenelementen in 4-dimensionalen Mannigfaltigkeiten, Math. Ann. 136 (1958), 156-172.

3. A. Haefliger and M. Hirsch, On the existence and classification of differentiable embeddings (to appear).

YALE UNIVERSITY 